\title{
A MONITORING SCHEME WITH IOT AND SENSOR EXPERTISE
}

\author{
Syeda Kausar Fatima \\ Research Scholar JNTUH, Prof., DCET, Hyderabad, Principal NSAKCET, \\ Prof., ECE Dept, JNTUH, India. \\ Dr. Syeda Gauhar Fatima \\ Research Scholar JNTUH, Prof., DCET, Hyderabad, Principal NSAKCET, \\ Prof., ECE Dept, JNTUH, India. \\ Dr. Syed Abdul Sattar \\ Research Scholar JNTUH, Prof., DCET, Hyderabad, Principal NSAKCET, \\ Prof., ECE Dept, JNTUH, India.

\section{Dr. Anita Sheela} \\ Research Scholar JNTUH, Prof., DCET, Hyderabad, Principal NSAKCET, \\ Prof., ECE Dept, JNTUH, India.
}

\begin{abstract}
Air quality has been largely affected by industrial activities, which have caused many health issues among people. Air pollutants levels can be measured using gas sensors. Internet of Things (IoT) technology can be used to remotely detect pollution. IOT devices can be used to control basic functions from anywhere around the world through the internet. The data gathered by such a system can be transmitted instantly to a web-based application to enable monitoring real time data and allow immediate risk management. The aim is to build a system which can be used to monitor the parameters in different environments. Here, we describe an entire Internet of Things (IoT) system that collects real-time data in specific locations. This real-time data collected is compared with a predetermined threshold. This data is sent to the concerned organization notifying them about the values exceeding the threshold if any and take necessary actions if needed.
\end{abstract}

Keywords Gas sensors, Pollution Monitoring, Global Positioning system (GPS), Internet of Things (IoT), Machine learning Algorithm.

Cite this Article: Syeda Kausar Fatima, Dr. Syeda Gauhar Fatima, Dr. Syed Abdul Sattar, and Dr. Anita Sheela, A Monitoring Scheme with Iot And Sensor Expertise, International Journal of Advanced Research in Engineering and Technology, 10(2), 2019, pp. 271-277.

http://iaeme.com/Home/issue/IJARET?Volume $=10 \&$ Issue $=2$ 


\section{INTRODUCTION}

Air pollution is a major issue affecting the atmosphere in various ways. Air pollution also causes many ill effects in animals, plants and human beings. Indoor air pollution is considered to be the most toxic kind of pollution. There are several different gases whose levels when exceeds some threshold value affects the atmospheric air quality. These gases are very harmful to human health and also the environment. Due to the different harmful gases there are atmospheric problems like global warming, acid rains and drastic climatic changesIn this paper, we describe a system which uses IoT technology to help in monitoring and analysing the air quality level by measuring the pollutants level in the atmosphere. The approach mainly focused here is to keep the people near industrial areas safe from the different threats caused by the air pollutants level by informing the official authorities about any violations against the threshold values for different gases. Pollution detectors are common in many environments like hospitals and other public places, but here the aim and objective are to that they must be placed within industrial regions and factories. Here the focus is on using technology for betterment of air quality while minimizing resources that are consumed for the process.

The Internet of Things is a technology in which physical devices are connected over a network to exchange real-time data. Data can be transmitted from one point to another without human involvement, in such a system. The IoT allows objects to be sensed or controlled remotely across existing network infrastructure. The Internet of things is applicable to many aspects in everyday life, whether it is at home, work, transport, or outdoors. Smart homes, healthcare systems, and wearable devices are all examples of IoT implementations.

A remotely accessible environment is an environment in which each appliance can be remotely accessed and controlled using software as an interface, which includes an Android application. Purification of air quality when the gas levels exceeds the thresholds can be done using air purifying devices. These devices can be controlled remotely using IoT technology where the control is given to the official authorities. When they are notified that the current pollutants level exceeding their thresholds, they can remotely switch on the purification devices setup. This helps in minimizing energy consumption since the purification device is switched on when necessary by remote controlling.

\section{PROPOSED SYSTEM}

The system to be developed is a wireless sensor network for monitoring air pollution. To achieve this system, the sensors are set at different locations where the pollution levels are to be monitored. The sensor values are read and noted in different conditions and timings of the day. This is done in order to collect data sets for the pollutant's values at different environmental locations. These datasets are used to select the threshold value for different gases and real time data collected is compared against these threshold values to say if there is any violation or threat to the air quality.

The gas sensors are connected to a microcontroller which is connected to a WIFI module. The output of the sensors is given to the Arduino board which contains the microcontroller and all the sensor outputs that is the real time data is sent to the cloud for storage. 


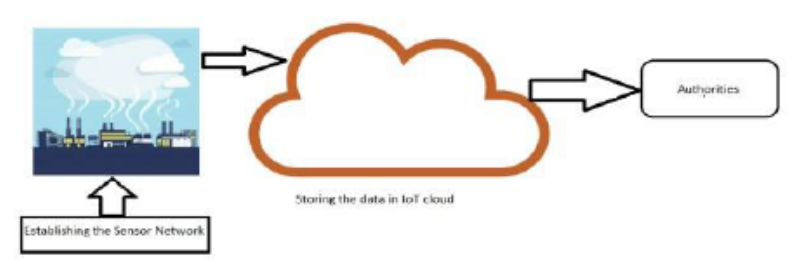

Figure 1 Proposed system model

Thus, the proposed system model consists of the following Arduino board consisting of the microcontroller, different gas sensors, gas sensors used are specific to the particular gases. WIFI module used is ESP 8266 which is used to connect the hardware that is the Arduino to the internet so that the real time data collected is sent to the cloud for storage in the cloud database. There is a global positioning system used so that the location also is sent as real time data and stored in the cloud. A purification device is also set up which can be controlled remotely using IoT.

\section{ARCHITECTURE}

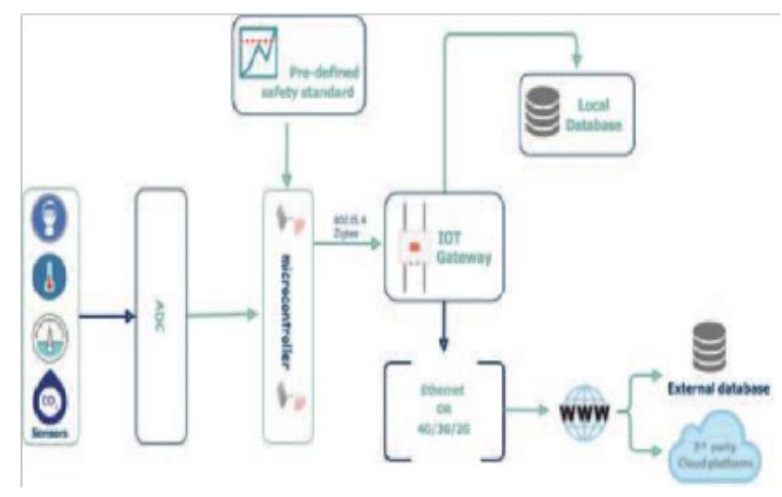

Figure 2 The design of the system

The figure shows the overall system architecture of environmental monitoring wireless sensor network system. Sensors are a major part in this system it is responsible for information or real time data.

\section{ARDUINO UNO}

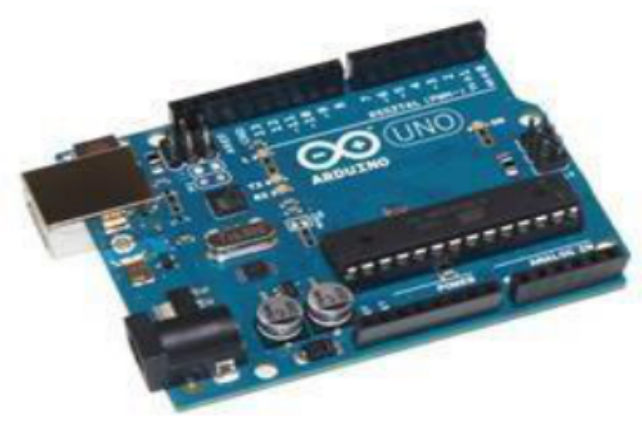

Figure 3 Arduino UNO board

Arduino is an open source computer hardware and software company, project, and user community that designs and manufactures single-board and microcontroller kits for building 
digital devices and interactive objects that can sense and control objects in the physical and digital world. Arduino board designs use a variety of microprocessors and controllers. The boards are equipped with sets of digital and analog input/output (I/O) pins that may be interfaced to various expansion boards or Breadboards (shields) and other circuits. The boards feature serial communications interfaces, including Universal Serial Bus (USB) on some models, which are also used for loading programs from personal computers. The microcontrollers are typically programmed using a dialect of features from the programming languages $\mathrm{C}$ and $\mathrm{C}++$. In addition to using traditional compiler toolchains, the Arduino project provides an integrated development environment (IDE) based on the Processing language project. This is the new Arduino Uno R3. In addition to all the features of the previous board, the Uno now uses an ATmega16U2 instead of the 8U2 found on the Uno (or the FTDI found on previous generations). This allows for faster transfer rates and more memory. No drivers needed for Linux or Mac (file for Windows is needed and included in the Arduino IDE), and the ability to have the Uno show up as a keyboard, mouse, joystick, etc.

\section{GAS SENSORS}

Different gas sensors are used to detect specific gases and the gas sensors used are MQ-135, MQ-5, MQ-4, MQ-8, MQ-9.

These are semiconductor sensors. The working principle of the semiconductor sensors is that they rely on a gas coming into contact with a metal oxide surface. The change in conductivity or resistivity is a linear and proportional relationship with the concentration of the gas coming in contact with the semi conducting material used. Tin dioxide is the most common material used in semiconductor sensors, and the electrical resistance in the sensor is decreased when it comes in contact with the monitored gas. This change in resistance is used to calculate the gas concentration.

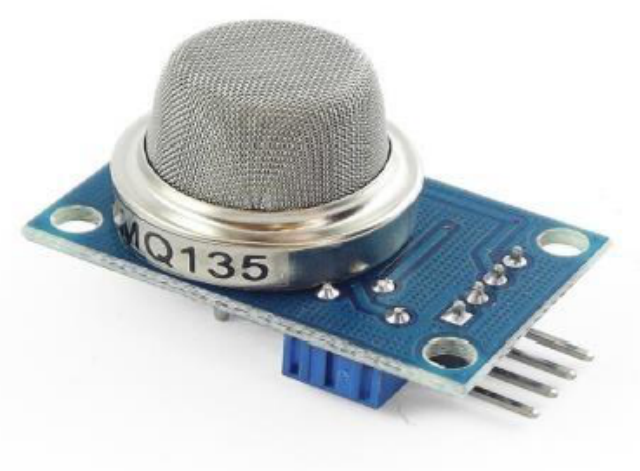

Figure 4 Semi-conductor Gas Sensor

MQ-135 is sensitive to ammonia, alcohol and benzene. MQ-5 is sensitive to LPG. MQ-4 is used to detect methane. MQ-8 is used to detect hydrogen gas. MQ-9 is sensitive to carbon monoxide gas. Due to the reason that the sensor must come in contact with the gas to detect it, semiconductor sensors work over a smaller distance than infrared point or ultrasonic detectors. All gas detectors must be calibrated on a schedule. Of the two form factors of gas detectors, portable gas detectors must be calibrated more frequently due to the regular changes in environment they experience. 


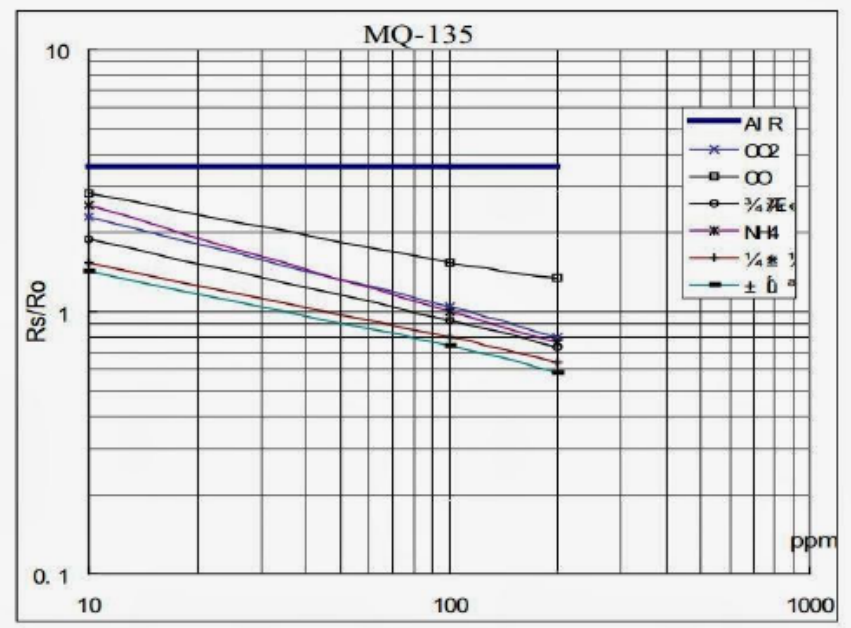

Figure 5 shows the typical sensitivity characteristics of the MQ-135 for several gases.

\section{WIFI MODULE- ESP8266}

The ESP8266 is a low-cost Wi-Fi microchip with full TCP/IP stack and microcontroller capability. This small module allows microcontrollers to connect to a Wi-Fi network and make simple TCP/IP connections.

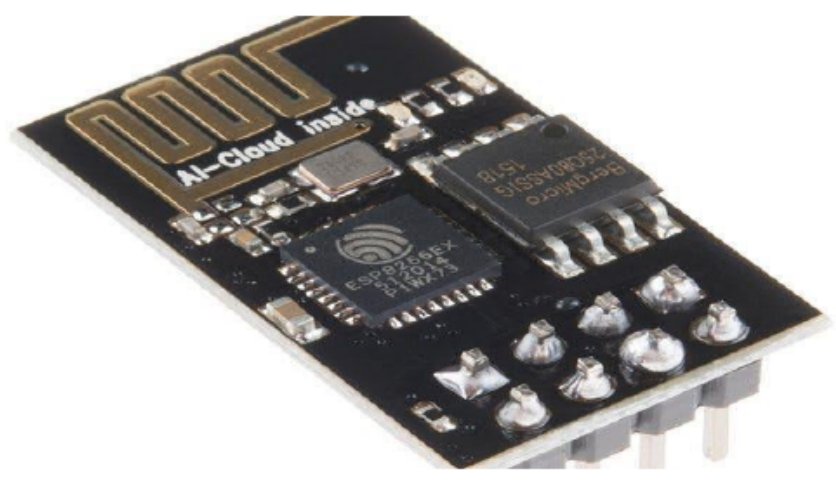

Figure 6 ESP8266, WiFi module

The processor used in the board is L106 32-bit RISC microprocessor core running at 80 MHz. The module has $32 \mathrm{KiB}$ instruction RAM, $32 \mathrm{KiB}$ instruction cache RAM, $80 \mathrm{KiB}$ user data RAM, 16 KiB ETS system data RAM. Espressif Systems released a software development kit (SDK) that allowed the chip to be programmed, removing the need for a separate microcontroller. There have been many official SDK releases. The other open source SDK is Arduino, which is a C++ based firmware. This core enables the ESP8266 CPU and its Wi-Fi components to be programmed like any other Arduino device. Ai Thinker Modules are the first series of modules made with the ESP8266 by the third-party manufacturer Ai-Thinker. They are collectively referred to as "ESP-xx modules". To form a workable development system they require additional components, especially a serial TTLto-USB adapter and an external 3.3-volt power supply.

\section{GPS NAVIGATION DEVICE}

It comprises of the GPS receiver and the antenna. GPS receiver is a device that is capable of receiving information from_GPS satellites. It then calculates the device's geographical position. Using suitable software, the device may display the position on a map, and it may offer directions. A GPS device can retrieve from the GPS system location and time information in all weather conditions, anywhere on or near the Earth. A GPS reception requires an 
unobstructed line of sight to four or more GPS satellites, ${ }^{[2]}$ and is subject to poor satellite signal conditions. GPS devices vary in sensitivity, speed, vulnerability to_multipath propagation, and other performance parameters.

High Sensitivity GPS receivers use large banks of correlators and digital signal processing to search for GPS signals very quickly.

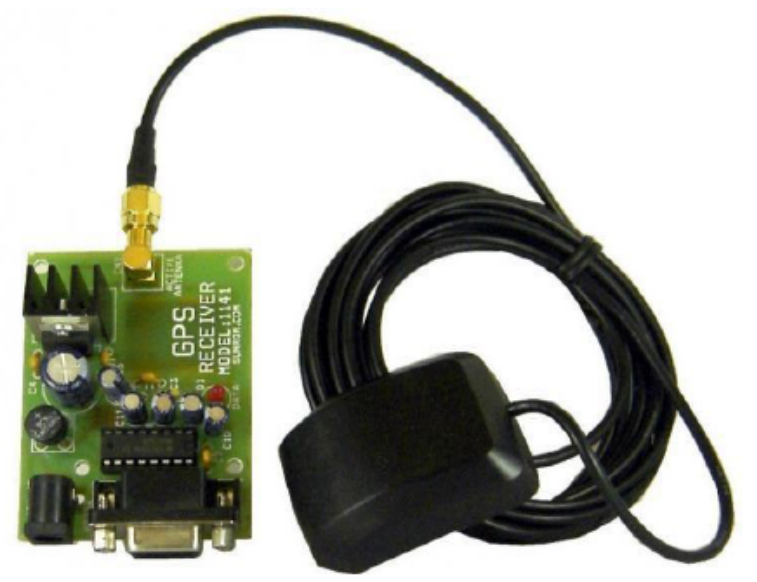

Figure 7 GPS navigation device

When GPS signals are weak, for example indoors, the extra processing power can be used to integrate weak signals to the point where they can be used to provide a position or timing solution. When indoor locations cannot be recognized due to week signals the antenna can be placed outside so that the location can be detected.

\section{EXPERIMENTAL OBSERVATIONS AND RESULTS}

The system implementation and hardware connections are shown in the figure. The analog signal from the sensors is converted to a digital signal where it is passed as an input to a microcontroller device, which compares the measured value with a threshold value. The result is then transmitted using the internet of things technology to a database on the cloud where the data is stored as required. The signal acquired from the sensors from different areas is compared with the predetermined values in the microcontroller. When the pollution is below the threshold, the sensors keep reading data continuously. When the pollution increases beyond the predetermined threshold, then the gathered data can be sent to the higher authorities for which appropriate actions can be taken by them.

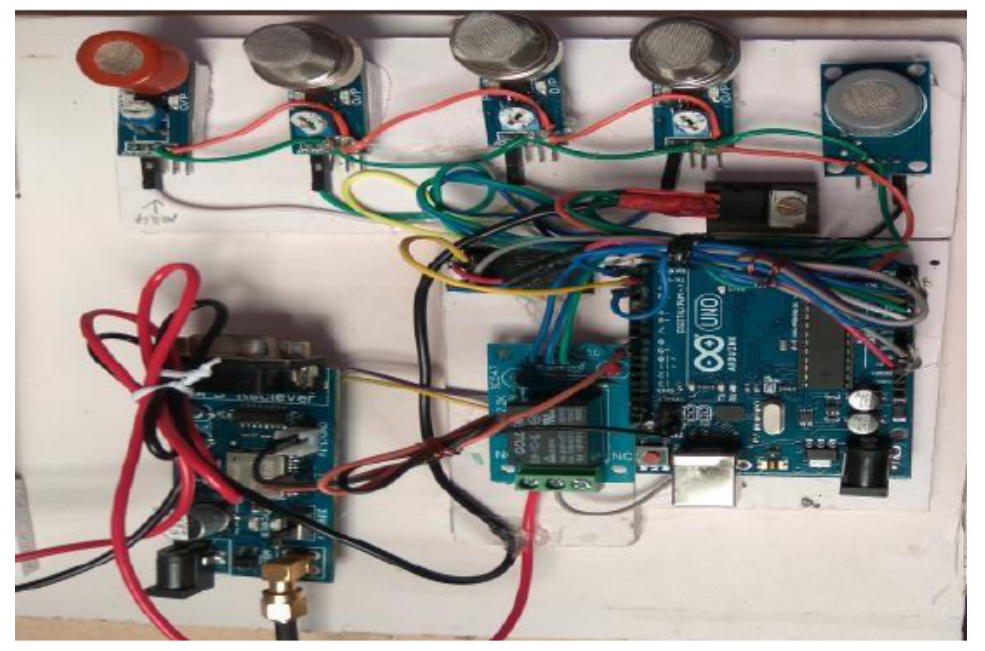

Figure 8 overall system design 


\section{CONCLUSION}

The aim of this paper is to build a system which monitors the pollution in an area and informs the authorities about the violation when the air pollutants exceeds the threshold value analyzed and stored in the comparison data set. The system also sends the location where this violation has occurred which helps the authorities for easy analysis. In this system sensors are implemented to detect the presence of undesired gases in the air of residential areas. The sensors provide continuous monitoring. Internet of Things technology is used to analyse the collected data for pollution. This system has low-cost, low power consumption, and easy to maintain.

\section{REFERENCES}

[1] Nupur Tyagi, "A Reference Architecture for IoT", International Journal of Computer Engineering and Applications, Volume X, Issue I, Feb,2016. pp. 19-24.

[2] Kennedy Okokpujie, Etinosa Noma-Osaghae, Odusami Modupe, Samuel John and Oluga Oluwatosin, A Smart Air Pollution Monitoring System, International Journal of Civil Engineering and Technology, 9(9), 2018, pp. 799-809.

[3] Rahol Jaikwad, "Internet of Things application areas, Smart Cities” INCON2016, Frankfurt, Jan. 2016.

[4] K. Raghava Rao, Monika Vallabhaneni, Srikanth Narayanaraju, Rajendra Kumar Jonnalagadda and Sunilkumar kanaparthi, Air Pollution Monitoring using Zigbee Based Wireless Sensor Networks, International Journal of Electronics and Communication Engineering \& Technology (IJECET), Volume 5, Issue 5, May (2014), pp. 56-63

[5] Deakin, Mark; Al Waer, Husam. "From Intelligent to Smart Cities". Journal of Intelligent Buildings International, Aug 2011, p.p. 140-152.

[6] Vu Ngoc Quang, Pham Thi Thanh Hoa and Nguyen Van Thinh, Determnation of Local Heght Using Global Positioning System with Low Price Receivers. International Journal of Civil Engineering and Technology, 9(8), 2018, pp. 1575-1582.

[7] Monitoring Pollution: Applying IoT to Create a Smart Environment-Anwar Alshamsi, Younas Anwar, Maryam Almulla, Mouza Aldohoori, Nasser Hamad, Mohammed Awad, International Conference, 2017.

[8] Real Time Ambient Air Quality Monitoring System Using Sensor Technology, Jyoti Sharma, Siby John, International Conference, 2017.

[9] Web Based Air Pollution Monitoring System (Air Pollution Monitoring Using Smart Phone), Shilpa R. Khodve, A. N. Kulkarni, International Journal of Science and Research, 2014. 